\title{
Integrated care at home reduces unnecessary hospitalizations of community- dwelling frail older adults: a prospective controlled trial
}

Laura Di Pollina ${ }^{1 *}$, Idris Guessous ${ }^{1,2^{*}}$, Véronique Petoud ${ }^{4}$, Christophe Combescure ${ }^{3}$, Bertrand Buchs ${ }^{5}$, Philippe Schaller ${ }^{6}$, Michel Kossovsky ${ }^{1}$ and Jean-Michel Gaspoz ${ }^{1}$

\begin{abstract}
Background: Care of frail and dependent older adults with multiple chronic conditions is a major challenge for health care systems. The study objective was to test the efficacy of providing integrated care at home to reduce unnecessary hospitalizations, emergency room visits, institutionalization, and mortality in community dwelling frail and dependent older adults.

Methods: A prospective controlled trial was conducted, in real-life clinical practice settings, in a suburban region in Geneva, Switzerland, served by two home visiting nursing service centers. Three hundred and one communitydwelling frail and dependent people over 60 years old were allocated to previously randomized nursing teams into Control $(N=179)$ and Intervention $(N=122)$ groups: Controls received usual care by their primary care physician and home visiting nursing services, the Intervention group received an additional home evaluation by a community geriatrics unit with access to a call service and coordinated follow-up. Recruitment began in July 2009, goals were obtained in July 2012, and outcomes assessed until December 2012. Length of follow-up ranged from 5 to 41 months (mean 16.3). Primary outcome measure was the number of hospitalizations. Secondary outcomes were reasons for hospitalizations, the number and reason of emergency room visits, institutionalization, death, and place of death.

Results: The number of hospitalizations did not differ between groups however, the intervention led to lower cumulative incidence for the first hospitalization after the first year of follow-up $(69.8 \%, \mathrm{Cl} 59.9$ to 79.6 versus $87 \cdot 6 \%, \mathrm{Cl} 78 \cdot 2$ to $97 \cdot 0 ; p=.01$ ). Secondary outcomes showed that the intervention compared to the control group had less frequent unnecessary hospitalizations (4.1\% versus $11.7 \%, p=.03)$, lower cumulative incidence for the first emergency room visit, $8.3 \%, \mathrm{Cl} 2.6$ to 13.9 versus $23.2 \%, \mathrm{Cl} 13.1$ to $33.3 ; p=.01$ ), and death occurred more frequently at home (44.4 versus $14.7 \% ; p=.04$ ). No significant differences were found for institutionalization and mortality.

Conclusions: Integrated care that included a home visiting multidisciplinary geriatric team significantly reduced unnecessary hospitalizations, emergency room visits and allowed more patients to die at home. It is an effective tool to improve coordination and access to care for frail and dependent older adults.
\end{abstract}

Trial registration: Clinical Trials.gov Identifier: NCT02084108. Retrospectively registered on March 10 2014.

Keywords: Aged, Community based interventions, Home care, Chronic disease, Palliative care

\footnotetext{
* Correspondence: I.dipollina@noshaq.ch; idris.guessous@hcuge.ch

${ }^{1}$ Division of Primary Care Medicine, Department of Community Medicine,

Primary Care and Emergency Medicine, Geneva University Hospitals, 1, avenue

Calas, Geneva 1206, Switzerland

Full list of author information is available at the end of the article
} 


\section{Background}

Major prolongation of life expectancy has led to a rapid increase in the number of older adults with multiple chronic conditions and dependency [1]. Improving the ability of health care systems to provide high-quality cost-effective health care for this population is a major challenge. It will require a paradigm shift from episodic, short-term interventions characteristic of acute care, to long-term, comprehensive care [2]. Furthermore, the needs and disabilities of frail elders are frequently not identified [3-5]. Fragmentation and discontinuity of care within and between health and social sectors results not only in frequent and unnecessary hospitalizations and emergency room visits, but also in premature nursing home placement with increased health care costs [6]. A large proportion of these patients die unnecessarily in hospital [7-10].

There has been an increasing interest to test whether integrated models of care can improve health outcomes and to shift service utilization from institutions toward communities. Enabling older adults to remain at home has become a priority of government policy in many countries [11].

Depending on the level of collaboration between administrative, financial, and service sectors, three models of integration have been described [12, 13] linkage, coordination and full integration, with a continuum in the degree of collaboration ranging from informal to structured, with all health, social, and supportive services included under a single roof.

While some integrated models have improved health status, the results of satisfaction and utilization of resources are inconsistent. This may be explained, in part, by differences in settings, study design, interventions, length of follow-up, financing, and measured outcomes [14-22]. Furthermore, results from trials that evaluated the effect of in-home geriatric assessment and preventive home visitation programs have also been variable [23-36]. Implementation of these programs outside demonstration projects remains a challenge, particularly in a multi-payer system.

We conducted a prospective controlled study performed in real-life clinical practice settings to evaluate the efficacy of formally coordinating existing resources: 2 home visiting nursing service centers (HVNS) and a community geriatric unit (CGU) that included a physician to perform in-home multidimensional geriatric assessment, and a 24h/ 7 days a week call service for frail older adults. We hypothesized that this approach could decrease the number of hospitalizations, decrease or delay unnecessary hospitalizations, emergency room visits, and institutionalization, as well as increase the probability of respecting care goals of patients with advanced illness who wish to remain at home.

\section{Methods}

\section{Study design and setting}

This prospective controlled trial was performed in two neighboring communities of the Canton of Geneva: Onex (population 17,942) and Bernex, (population 9,772). The socioeconomic status of this population is homogeneous, and $16 \%$ are over 65 years old. Two home visiting nursing service centers (HVNS) serve this region, each staffed with two nursing teams (NT) of similar size and composition (eight nurses per team), who provide home visits to patients. Patients are routinely assigned to a NT depending on their place of residence.

\section{Participants}

Participant recruitment began in July 2009 in the Onex HVNS center. Because of difficulty in obtaining recruitment goals the Bernex HVNS center was invited to participate in June 2011. Recruitment goals were obtained in July 2012, and outcomes assessed until December 2012. Consecutive patients 60 years and older who were identified as frail were eligible if followed by a primary care physician (PCP), who had prescribed HVNS services. On the first home visit, nurses routinely performed an initial assessment using items of the Resident Assessment Instrument-Home Care (RAI-HC) [37, 38].

Inclusion criteria were presence of frailty, as defined by one of four alarms or risk factors (impaired cognition, falls, social isolation, or frailty of the informal caregiver support) detected by the RAI-HC. If one risk factor was present, the research nurse calculated a frailty score using the Contact Assessment tool (derived from the InterRAI set of tools) [23]. The nine item frailty measure includes four activities of daily living (bathing, grooming, dressing, walking), two social environment items (living alone and absence of informal caregiver) and three health status items (cognitive impairment, perceived health as poor, and shortness of breath at rest or while performing daily activities). One point is attributed to each affected item, and scores between 1 and 5 reflect mild to moderate frailty, scores $\geq 6$ identify severe frailty [39]. Exclusion criteria were patients who did not meet frailty criteria, could not speak French, or were unable to give consent.

\section{Sample size calculation and randomization}

A sample size of 300 (150 per group) was planned based on a $5 \%$ alpha error (two-sided) and a power of $80 \%$ to detect a difference in hospitalization rates of $13 \%$ per year. This difference was based on a previous metaanalysis of controlled studies that utilized comprehensive geriatric assessment [25]. The expected rate of hospitalization was $30 \%$.

Because of the organization of healthcare delivery, (home visits by nurses), it was not possible to randomize 
patients individually. In real life clinical setting, each nursing team is responsible for following patients in a predetermined sector, by patient address. To avoid a bias in the allocation of sectors in the control or intervention arm, nurses were randomly assigned in two teams of similar size and composition (eight nurses per team), one team for each arm of the trial. Participants were sequentially allocated into one of the NT groups. Using a random number generator, each NT was designated by a number ( 1 to 4 ) and the first four random numbers generated were allocated to the corresponding NT. It was a priori decided that the highest number in each site would be randomized to the intervention and the other to the control group. Blinding was not possible due to the knowledge of the intervention.

\section{Control group}

Participants $(N=179)$ continued care with their PCP who prescribed services by the HVNS, which include home visits by nurses and nurses' aides one to three times a day depending on patients' needs (e.g., administration of medication, measurement of vital signs and glycaemia, wound care, support in activities of daily living) and /or technical support for in-home hospitalization. No formal case management was provided. As not all PCPs perform home visits, participants who required emergency service after office hours were usually instructed to contact a physician-oncall emergency service or go to an emergency room.

\section{Intervention group}

Participants $(N=122)$ were followed by their PCP, the intervention NT, and additionally provided with inhome geriatric assessment by the community geriatrics unit (CGU) doctor in the following domains: cognition (Mini-Mental State Exam [40] and clock drawing test), mood (Geriatric Depression Scale [41]) functional status (basic [42] and instrumental activities of daily living, [43] gait (timed up and go [44] and semi tandem stand, [45] nutrition (Mini-Nutritional Assessment (short form), [46] pain (visual analogue scale), medication review and adherence. The results were transmitted in writing to the PCP and NT with recommendations, and, in the event of complex issues, meeting between the CGU and NT were organized. Participants and NT received written instructions to first contact the PCP in case of an emergency; if unavailable, the CGU that provided a $24 \mathrm{~h} /$ 7 days a week medical call service was contacted. The CGU home intervention team included doctors, physical and occupational therapists, psychologists, dieticians and social workers. A day hospital is also part of provided services [47].

\section{Measures}

Data were collected from patient computerized medical and nursing records. The primary outcome measure was the number of hospitalizations. Secondary outcomes were the reason of hospitalization and the number and reason of emergency room visits, institutionalization, death, and place of death.

Reasons for hospitalizations included falls, fractures, acute medical problems, psychiatric problems, or social reasons (unnecessary). Reasons for emergency room visits included falls, acute medical, or psychiatric problems and social reasons (unnecessary). Reason and number of consultations for an acute problem (phone or home visit) by the CGU, PCP, or other emergency service were also collected.

Unnecessary hospitalizations were identified by chart review by the research nurse and defined as those occurring in the absence of an acute medical problem and that could have been handled by a general practitioner or home care program.

\section{Statistical analysis}

Descriptive results are reported by counts and percentages or by means and standard deviations (sd). Characteristics were compared using the Student test, Chi-square or Fisher exact tests. Primary and secondary outcomes were compared in intention-to-treat by using survival analyses. For mortality, the Kaplan-Meier survival estimator, log-rank test and Cox regression model were used. As death was a competing event for the other outcomes, competing risk models were used [48, 49]. The cumulative incidences and the hazard ratio were obtained with the package cmprsk for $\mathrm{R}$ version 3.0.1. All reported hazard ratios were adjusted to NT and tests for comparison of cumulative incidence over three-year' follow-up were stratified by NT. The proportionality of hazards was graphically inspected (plots of the complementary log-log functions of survival). When hazards were not proportional, an interaction between the intervention's effect and the time was added in the model: the estimates of hazard ratio (HR) could be different between the first year of follow-up and after the first year. The cumulative incidences were compared at one, two, and three years using a Wald test. Two-sided $p$-values less than 0.05 were considered significant. All analyses were performed using S-Plus 8.0 for Windows (Insightful Corp., Seattle, WA) and R version 3.0.1 (R Development Core Team. R: A Language and Environment for Statistical Computing. Vienna, Austria: R Foundation for Statistical Computing; 2010).

\section{Results}

Enrollment, allocation and follow-up are described in Fig. 1. Two hundred and twenty-three participants were 


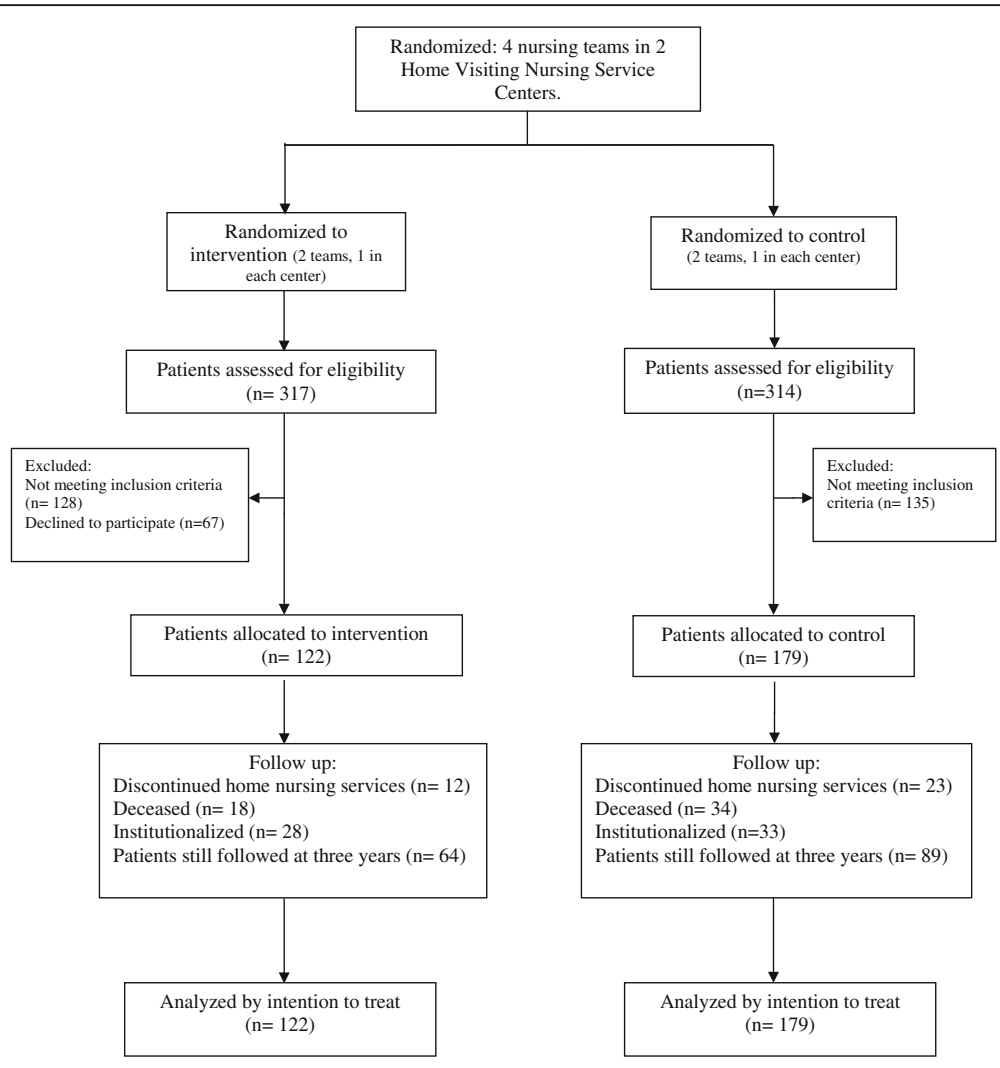

Fig. 1 Flow chart

recruited in the first two years, 78 in the last 12 months and 4 patients in the last 6 months. $52.5 \%$ of subjects in the intervention group and $49.7 \%$ in the control group were still in the study at the end of data collection (December 15 2012). Length of follow-up ranged from 5 to 41 months with a mean of 16.3 months and is reported in Table 1. Baseline characteristics are shown in Table 2 and were similar between groups.

\section{Hospitalizations}

Fifty nine percent of participants in the intervention group and sixty percent of participants in the control group had at least one hospitalization (Table 3). The cumulative incidence for a first hospitalization (Fig. 2), was not significantly lower in the intervention than in the control group $(p=0.24)$. However, a difference in favor of the intervention group appeared after the first year (three-year cumulative incidence $69.8 \%$ in the intervention group and $87.6 \%$ in the control group, $p=0.01$; see Table 3). In a post-hoc analysis, the HR was 0.94 (95\%CI 0.67 to $1.31, p=0.71)$ over the first year and $0.48(95 \% \mathrm{CI}$ 0.24 to $0.95, p=0.04$ ) over the second and third year of follow-up. The HR over the three year follow-up period was $0.82(95 \%$ CI 0.61 to $1.11, p=0.21)$

Unnecessary hospitalizations for social reasons were significantly less frequent in the intervention group ( $4.1 \%$ versus $11.7 \%, p=0.03$ ) (Table 3 ). Length of hospital stay was lower, but not significantly, in the intervention than in the control group (37.5 versus 51.0 days $p=0.18$ ).

\section{Emergency room visits}

Eight (6.6\%) participants in the intervention and 26 $(14.5 \%)$ in the control group had at least one emergency

Table 1 Description of length of follow-up and status at end of study

\begin{tabular}{|c|c|c|c|c|}
\hline & \multicolumn{2}{|c|}{ Intervention $(n=122)$} & \multicolumn{2}{|c|}{ Control $(n=179)$} \\
\hline & $N(\%)$ & Median follow-up in days [IQR] & $N(\%)$ & Median follow-up in days [IQR] \\
\hline Death & $18(14.8)$ & $294(116 ; 555)$ & $34(19.0)$ & $298(150 ; 505)$ \\
\hline Institutionalization & $28(23.0)$ & $412(203 ; 818)$ & $33(18.4)$ & $401(226 ; 619)$ \\
\hline Withdrawal & $12(9.8)$ & $148(41 ; 340)$ & $23(12.8)$ & $176(78 ; 337)$ \\
\hline Still in study & $64(52.5)$ & $592(308 ; 910)$ & $89(49.7)$ & $435(291 ; 599)$ \\
\hline
\end{tabular}


Table 2 Baseline demographic and clinical characteristics

\begin{tabular}{|c|c|c|c|}
\hline & $\begin{array}{l}\text { Intervention } \\
(N=122)\end{array}$ & $\begin{array}{l}\text { Control } \\
(N=179)\end{array}$ & $P$ value \\
\hline \multicolumn{4}{|l|}{ Baseline characteristics } \\
\hline Female & 78/122 (63.9\%) & $120 / 179(67.0 \%)$ & 0.66 \\
\hline Age & & & 0.99 \\
\hline $60-79$ & $49(40.2 \%)$ & 70 (39.3\%) & \\
\hline $80-89$ & $56(45.9 \%)$ & $82(46.1 \%)$ & \\
\hline $90-100$ & $17(13.9 \%)$ & $26(14.6 \%)$ & \\
\hline Mean (sd) & $81.8(8.2)$ & $81.9(8.2)$ & 0.85 \\
\hline Live alone & $32 / 121(26.4 \%)$ & $57 / 173(32.9 \%)$ & 0.29 \\
\hline $\mathrm{ADL}(0-6)$, mean $(\mathrm{sd})$ & $1.2(1.6)$ & $1.1(1.4)$ & 0.52 \\
\hline IADL (0-8), mean (sd) & $5.7(1.8)$ & $5.4(2.0)$ & 0.23 \\
\hline Perceived health as poor & $52 / 104(50.0 \%)$ & $65 / 161(40.4 \%)$ & 0.16 \\
\hline Psychotropic medications & 76/112 (67.9\%) & 93/137 (67.9\%) & 0.90 \\
\hline $\begin{array}{l}\text { Analgesics (opioid + non } \\
\text { opioid) }\end{array}$ & $67 / 115(58.3 \%)$ & 79/143 (55.2\%) & 0.72 \\
\hline $\mathrm{BMI}<21$ & 25/107 (23.4\%) & 29/135 (21.5\%) & 0.85 \\
\hline \multicolumn{4}{|l|}{ Alarms (RAI-HC instrument) } \\
\hline $\begin{array}{l}\text { Number of alarms (range } \\
1-4 \text { ) }\end{array}$ & $2.1(0.9)$ & $2.1(0.9)$ & 0.59 \\
\hline Cognition & $61 / 122(50.0 \%)$ & $88 / 179(49.2 \%)$ & 0.98 \\
\hline Falls & $89 / 122(73.0 \%)$ & $132 / 179(73.7 \%)$ & 0.98 \\
\hline Social isolation & 65/122 (53.3\%) & $87 / 179(48.6 \%)$ & 0.50 \\
\hline $\begin{array}{l}\text { Frailty of informal } \\
\text { caregiver }\end{array}$ & 46/122 (37.7\%) & $66 / 179(36.9 \%)$ & 0.98 \\
\hline \multicolumn{4}{|l|}{ CA SCORE } \\
\hline $\begin{array}{l}1-5 \text { (mild-moderate } \\
\text { frailty) }\end{array}$ & $92 / 122(75.4 \%)$ & 135/179 (75.4\%) & 0.89 \\
\hline 6-9 (severe frailty) & $30 / 122(24.6 \%)$ & $44 / 179(24.6 \%)$ & \\
\hline \multicolumn{4}{|l|}{ Diagnoses } \\
\hline Cardiac disease & $51 / 113(45.1 \%)$ & $54 / 159(34.0 \%)$ & 0.08 \\
\hline Degenerative joint disease & $59 / 113(52.2 \%)$ & $97 / 160(60.6 \%)$ & 0.21 \\
\hline Diabetes & 28/109 (25.7\%) & $34 / 153$ (22.2\%) & 0.61 \\
\hline $\begin{array}{l}\text { Obstructive pulmonary } \\
\text { disease }\end{array}$ & $27 / 110(24.5 \%)$ & $27 / 156(17.3 \%)$ & 0.20 \\
\hline Cancer & 26/112 (23.2\%) & $39 / 155$ (25.2\%) & 0.82 \\
\hline Depression & 46/108 (42.6\%) & $55 / 158(34.8 \%)$ & 0.25 \\
\hline Dementia & $30 / 110(27.3 \%)$ & $39 / 156$ (25.0\%) & 0.78 \\
\hline Stroke & 19/105 (18.1\%) & 28/153 (18.3\%) & 0.90 \\
\hline Chronic renal failure & 19/108 (17.6\%) & 16/152 (10.5\%) & 0.14 \\
\hline Urinary incontinence & 47/121 (38.8\%) & $57 / 176(32.4 \%)$ & 0.31 \\
\hline Chronic pain & 55/87 (63.2\%) & 75/111 (67.6\%) & 0.62 \\
\hline Visual impairment & $52 / 110(47.3 \%)$ & $63 / 157(40.1 \%)$ & 0.30 \\
\hline
\end{tabular}

Results are reported by counts and percentages or by means and standard deviation (sd)

Abbreviations: $A D L$ activities of daily living (score reported is number of deficient activities), IADL instrumental activities of daily living (score reported is number of deficient activities), RAI-HC Resident Assessment Instrument-Home Care, CA Contact assessment score room visit, $p=0.04$ (Table 3 ). The three-year cumulative incidences were $8.3 \%$ in the intervention and $23.2 \%$ in the control group, $p=0.01$ (Fig. 2). The HR over the entire follow-up period for emergency room visits was 0.43 ( $95 \%$ CI 0.19 to $0.94, p=0.04$ ).

\section{Institutionalization}

No difference was found ( $p=0.70$; see Fig. 2 and Table 3 ), with institutionalization for $28(23 \%)$ in the intervention and $33(18 \%)$ in the control group. HR was 1.05 (95\% CI 0.63 to $1.79, p=0.84$ ).

\section{Mortality}

Eighteen (14.8\%) participants in the intervention and 34 (19.1\%) in the control group died. The cumulative mortality was lower in intervention than in control group but not significantly $(p=0.06)$. A difference in mortality seemed to appear after the first year in favor of the intervention group but was not statistically significant (Fig. 2, Table 3). The HR over the entire follow-up was 0.58 ( $95 \%$ CI 0.32 to $1.05, p=0.07$ ). Death occurred significantly more often at home in the intervention group (44.4 versus $14.7 \%, p=0.04$; Table 3 ).

\section{Home consultations for an acute medical problem}

Ninety-seven participants in the intervention group (79.5\%) presented an acute problem leading to at least one urgent consultation by phone $(34.9 \%)$ or home visit (65.1\%) by the CGU doctor ( $60.3 \%$ of the time) or other emergency services. Ninety-five participants in the control group $(53.1 \%)$ received at least one urgent consultation, either by telephone $(47.7 \%)$ or through a home visit $(52.3 \%)$ by their PCP (58.4\% of the times) or another physician-on-call emergency service. On average, participants in the intervention received four times more consultations (telephone or home visit) than the control group (6.3 versus 1.6 per patient, $p<0.001$ ). Home visits resulted in a hospitalization in $8.7 \%$ of the cases in the intervention and in $19 \%$ in the control group $(p=<0.001)$.

\section{Discussion}

This prospective controlled trial showed that formally coordinating existing resources of the public and private sectors for frail and dependent-community dwelling older adults reduced the rate of hospitalizations after the first year, decreased unnecessary hospitalizations, lowered the rate of emergency room visits after the first year, and increased the proportion of patients dying at home. We did not find differences in the number of total hospitalizations or rates of institutionalization or mortality.

Few studies have included round-the-clock access to a geriatrics team. One such trial [21] included a 22-month 
Table 3 Main and secondary outcomes

\begin{tabular}{|c|c|c|c|}
\hline & $\begin{array}{l}\text { Intervention } \\
(n=122)\end{array}$ & $\begin{array}{l}\text { Control } \\
(n=179)\end{array}$ & $P$ \\
\hline \multicolumn{4}{|l|}{ Hospitalizations } \\
\hline No hospitalization ${ }^{\mathrm{b}}$ & 50/122 (41.0\%) & 73/179 (40.1\%) & \\
\hline One to three hospitalizations ${ }^{\mathrm{b}}$ & $66 / 122(54.1 \%)$ & 95/179 (53.1\%) & \\
\hline Four or more hospitalizations ${ }^{b}$ & $6 / 122(4.9 \%)$ & $11 / 179(6.1 \%)$ & \\
\hline One-year rate of first hosp. ${ }^{a, b}$ & 53.6 (43.9 to 63.3$)$ & 55.4 (47.0 to 63.8) & 0.78 \\
\hline Two-year rate of first hosp. ${ }^{a, b}$ & 67.8 (58.1 to 77.4$)$ & 82.3 (72.6 to 92.1) & 0.04 \\
\hline Three-year rate of first hosp. ${ }^{\mathrm{a}, \mathrm{b}}$ & 69.8 (59.9 to 79.6$)$ & 87.6 (78.2 to 97.0$)$ & 0.01 \\
\hline Length of stay (days) ${ }^{c}$ & 37.5 (18.8 to 84.0$)$ & 51.0 (26.3 to 93.5$)$ & 0.18 \\
\hline Reasons for hospitalizations ${ }^{d}$ & & & 0.03 \\
\hline Falls & 28/122 (23.0\%) & 29/205 (14.1\%) & 0.06 \\
\hline Fracture & 6/122 (4.9\%) & 8/205 (3.9\%) & 0.20 \\
\hline Acute medical problem & $80 / 122(65.6 \%)$ & 132/205 (64.4\%) & 0.92 \\
\hline Psychiatric problem & $3 / 122(2.5 \%)$ & 12/205 (5.9\%) & 0.18 \\
\hline Unnecessary & $5 / 122(4.1 \%)$ & $24 / 205(11.7 \%)$ & 0.03 \\
\hline \multicolumn{4}{|l|}{ Emergency room visit (ERV) } \\
\hline At least one ERV & $8 / 122(6.6 \%)$ & $26 / 179(14.5 \%)$ & 0.04 \\
\hline One-year rate of first ERV ${ }^{a}$ & 8.3 (2.6 to 13.9$)$ & 13.4 (7.8 to 19.0$)$ & 0.21 \\
\hline Two-year rate of first ERV ${ }^{a}$ & 8.3 (2.6 to 13.9$)$ & 17.8 (10.3 to 25.2$)$ & 0.045 \\
\hline Three-year rate of first ERV ${ }^{a}$ & $8.3(2.6$ to 13.9$)$ & $23.2(13.1$ to 33.3$)$ & 0.01 \\
\hline Reasons for consultation ${ }^{e}$ & & & 0.28 \\
\hline Falls & $5 / 8(62.5 \%)$ & 10/34 (29.4\%) & 0.11 \\
\hline Unnecessary & $0 / 8(0.0 \%)$ & $8 / 34(23.5 \%)$ & 0.32 \\
\hline Acute medical problem & $3 / 8(37.5 \%)$ & 15/34 (44.1\%) & 1 \\
\hline Psychiatric & $0 / 8(0.0 \%)$ & $1 / 34(2.9 \%)$ & 1 \\
\hline \multicolumn{4}{|l|}{ Institutionalization } \\
\hline One-year rate of placement ${ }^{a}$ & $11.2(5.1$ to 17.2$)$ & $10.3(5.3$ to 15.4$)$ & 0.83 \\
\hline Two-year rate of placement ${ }^{a}$ & 21.6 (12.9 to 30.4$)$ & 28.2 (18.4 to 37.9$)$ & 0.33 \\
\hline Three-year rate of placement ${ }^{a}$ & 39.4 (25.1 to 53.6$)$ & 31.8 (21.3 to 42.3$)$ & 0.40 \\
\hline \multicolumn{4}{|l|}{ Mortality } \\
\hline One-year mortality ${ }^{a}$ & $9.4(3.8$ to 15.1$)$ & $15.0(8.9$ to 21.1$)$ & 0.19 \\
\hline Two-year mortality ${ }^{a}$ & 17.9 (8.9 to 27.0$)$ & 33.7 (23.1 to 44.3$)$ & 0.03 \\
\hline Three-year mortality ${ }^{a}$ & 20.4 (10.4 to 30.3$)$ & 33.7 (23.1 to 44.3$)$ & 0.07 \\
\hline \multicolumn{4}{|l|}{ Place of death } \\
\hline At home & 8/18 (44.4\%) & $5 / 34(14.7 \%)$ & 0.04 \\
\hline
\end{tabular}

${ }^{a}$ cumulative incidences are expressed in percentage and reported with $95 \%$ confidence intervals

${ }^{b}$ the rate of first hospitalization rate were assessed using Kaplan-Meier's approach to account for varying length of follow-up across patients, while the reported percentages of with no, one to three and four or more hospitalizations did not account for length of follow-up and were only descriptive statistics

includes hospitalization in internal medicine and geriatrics and rehabilitation

${ }^{d}$ percentages of the number of hospitalizations, epercentages of the number of visits

follow-up and showed a 50\% reduction in hospital alternate level stays with no reduction in emergency room utilization, hospitalization or nursing-home placements.

Although we did not find a global reduction in hospitalizations over the follow-up period, the rate of hospitalizations was decreased already after the first year, suggesting that reduction in these rates may be related to length of follow-up. Unlike other studies that did not record the reason for hospitalization and emergency room visits, we were able to report that these events were mostly justified by an acute illness (most frequently an acute cardiac event, stroke, infections, falls, fractures, psychiatric and behavioral problems in demented patients as well as need for in-hospital palliative care), and, 

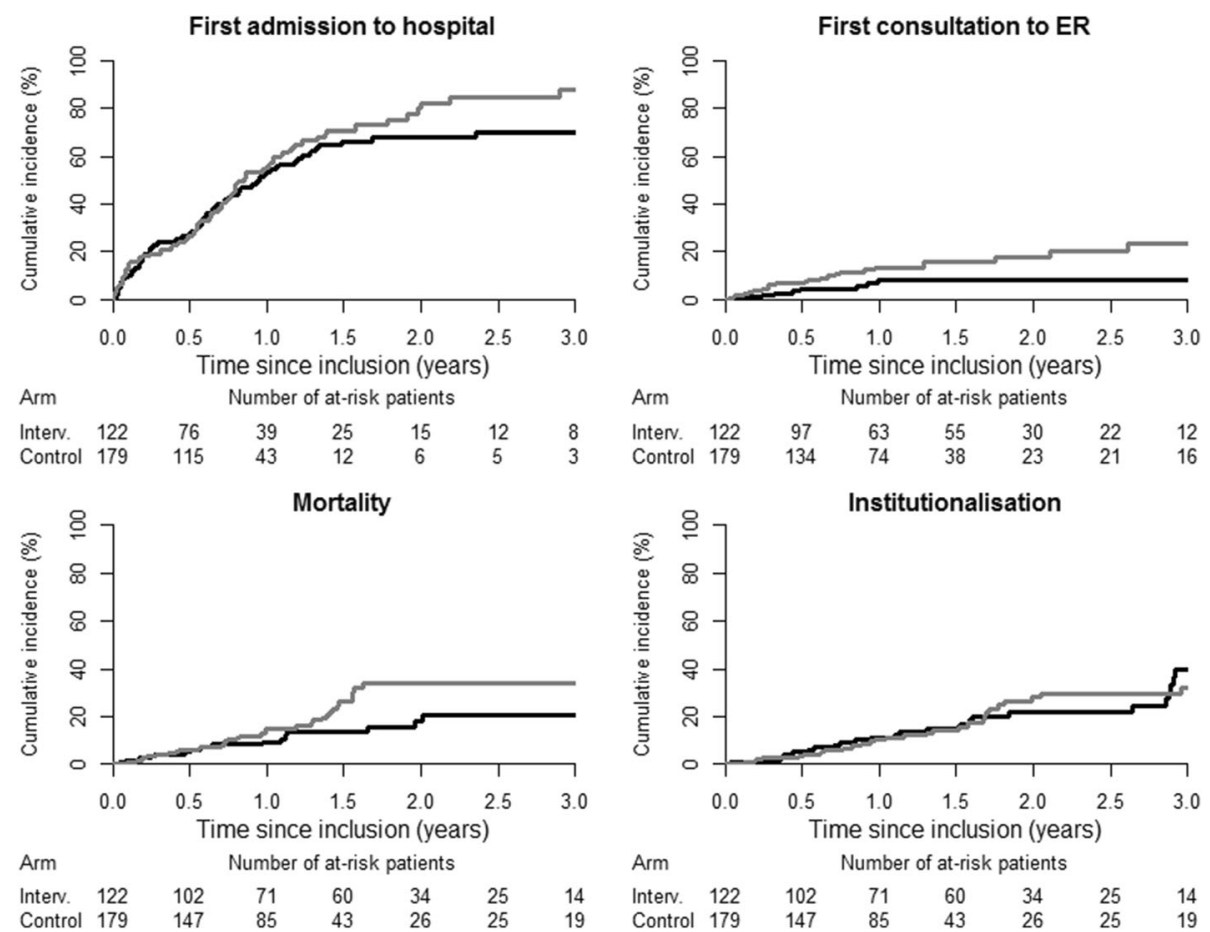

Fig. 2 Cumulative incidences for primary and secondary outcomes. First hospitalization rate was not significant different over the three year follow-up period (global $p=0.24)$. However, first hospitalization rate was significantly reduced at two $(p=0.04)$ and three years $(p=0.01)$. First emergency room visit was significantly reduced over the three year follow-up period (global $p=0.03$ ). No difference was found in institutionalization rates (global $p=0.70$ ). Mortality was not significantly reduced (global $p=0.06$ ) over the three year follow-up period. A significant difference appeared at two years $(p=0.03)$ with a trend at three years $(p=0.07)$ in favor of the intervention arm (see text for details)

more importantly, that unnecessary hospitalizations due to social reasons, such as lack or breakdown of the informal caretaker system, had decreased. In fact, the intervention arm received four times more consultations at home by the UGC for an acute problem that resulted in a hospitalization in a small number of the cases $(8.7 \%)$. Although it is possible that this quantitative difference alone may be responsible for some of the results, it is likely that qualitative changes and geriatric expertise were also important in ensuring better primary outcomes and allowing those who wished to die at home. These results suggest that improving collaboration and communication with rapid access to care in case of emergency or after office hours could avoid undesirable outcomes, since this also had a positive effect in the reduction of emergency room visits in the intervention group.

Several trials and meta-analyses have stated that the efficacy of comprehensive geriatric assessment is highly dependent on the number of visits, control over implementation of recommendations, and extended ambulatory follow-up [26-30]. Programs that include integrated models of care have been more likely to be effective $[17-23,35]$. Our study is consistent with these findings.
Many reviews of geriatric assessment have suggested to target primarily healthier older populations [33]. We were able to show several positive results of a simple integrated intervention in a particularly old and dependent group. Thus, our findings provide important evidence for the inclusion of highly dependent populations in integrated models of care.

No difference was found between groups in rates of institutionalization. This is consistent with a 2002 meta-analysis of home visit programs that showed reduced nursing-home placement only in high functioning older persons with multiple follow-up visits [30]. Institutionalization is also closely related to bed availability. And, it should be noted that our trial was carried out in a Swiss region with the lowest number of long-term care beds per capita and a long average wait for a nursing-home bed [50].

Although a global effect on mortality was not shown, the significant decrease at two years and the trend at three years suggests that mortality may have been postponed. Reduction of mortality has been reported in an earlier meta-analysis mostly in younger populations [30]. Our results are particularly striking in view of the heavy burden of disease and disability in our sample, and warrants confirmation in larger studies. 
Most importantly, the intervention had a marked positive effect on place of death, an issue not reported in most prior trials of in-home geriatric assessment, and reflects the capacity of integrated models to honor individuals' preferences and deliver quality end-of-life care. Although we considered "dying at home" as a desirable outcome, it should be acknowledged that some people with concrete palliative care needs prefer to be hospitalized before death, We found in our study that most of our patients declared their wish to die at home and were able to carry out this wish in slightly less than half of the intervention cases. This was a significant improvement compared to controls, but suggests that further efforts are needed to increase access to this option. A similar result was reported by the program of All-Inclusive Care for the Elderly (PACE), [9] although not evaluated by a controlled trial; it showed that $45 \%$ of participants died at home, compared to $20 \%$ in the general geriatric population.

The strengths of our approach include the integration of existing public (HVNS and CGU) and private (PCP) services, the availability of a multidisciplinary geriatrics team, including a round-the-clock medical call service, and a detailed analysis of the reasons for hospitalizations and emergency room visits, as well as place of death. Furthermore, as recommended in a large 2008 meta-analysis, [33] this study was conducted in a real clinical practice setting.

Limitations of our study include that patients were not randomized individually but only to nursing teams. Therefore, we cannot exclude that differences in outcomes between intervention and control arms are potentially caused by a confounding phenomenon. However, patients' characteristics were similar in both arms, in particular characteristics that captured frailty, hence the risk of a confounding phenomenon related to these characteristics is low. Furthermore, since the intervention and the control nursing teams worked in the same HVNS center, it was not possible to perform a doubleblind study and contamination between groups cannot be excluded. The research nurse who collected objective outcomes was independent and not a member of the HVNS center or the CGU and had no stake in the results of the study, further reducing the risk of introducing bias.

The study was initially designed to assess the cumulative incidence of first hospitalization at one year. However, due to lower than expected recruitment, the enrollment period was extended at the first site and a second HVNS center was added with a shorter follow-up than the first. Therefore, we analyzed data up to end of follow-up and also at one year. Although only half the subjects remained in the study upon its termination, most of the drop-outs were due to reached pre-determined end points, such as death or institutionalization, which reflects the severe morbidity burden of the studied population.

We have used a definition of frailty based on the RAI$\mathrm{HC}$ classification system, which has the advantage of being nursing based and is routinely administered on admission. It is, however, slightly different from the more medically-based Fried criteria [51] (for example weight loss and isometric hand strength are not included) and readers should take this into consideration when comparing our results to other studies.

Finally, we encountered several difficulties and temporary interruptions in recruitment during the study period. For example, the Onex center moved their offices to a nearby location at the end of 2010. The Bernex center transitioned to a computerized record system in the summer of 2011. A 5 month maternity leave of the research nurse in 2010 and need to find a replacement was an added reason for recruitment delay. All these events show that promoters of complex interventions in everyday practice should be aware of the possible emergence of unexpected environmental changes.

\section{Conclusions}

This three-year trial showed effectiveness of an integrated care approach that included in-home geriatric assessments performed by a physician, long-term coordinated follow-up, and availability of a round-the-clock geriatric call service, for the care of frail and dependent elderly. This approach led to the following outcomes: a reduction in hospitalization rate after the first year; a reduction in unnecessary hospitalizations due to social problems; a global reduction in emergency room visits; and, an increase in the proportion of patients who could die in their own home. These are important quality-of-life issues for older adults with progressive chronic illness.

We expect these results may be reproducible in other systems since the study was performed in a real life clinical practice setting and utilized existing resources. Finally, these results suggest the need to expand and implement integrated care models, including multidisciplinary geriatrics teams who can provide home visits and call services as a tool to improve long-term, comprehensive care for highly dependent community dwelling older adults.

\footnotetext{
Abbreviations

CGU: Community geriatrics unit; HVNS: Home visiting nursing service; NT: Nursing team; PCP: Primary care physician; RAI-HC: Resident assessment Instrument-Home Care

Acknowledgements

We wish to thank the management of IMAD (HVNS), as well as the nurses and nurses' aides of that establishment - without whom care for the frail elderly would not be possible at home - the private practitioners, the geriatrics unit team, the patients, and their families. Special acknowledgement is given to Aline Folliet RN and Suzanne Roux-Stuber RN, research nurses, for patient enrollment, and data collection, and to Nancy S Foldi PHD for manuscript review and editing.
} 


\section{Funding}

Geneva University Hospitals Quality Fund and the Edmond J Safra Foundation. Funders' role: Funding was used to hire the research nurse for data collection. The sponsors had no other role in the study.

\section{Availability of data and materials}

The datasets during and/or analyzed during the current study are available from the corresponding author on reasonable request.

\section{Authors' contributions}

LDP principal investigator; was involved in the study design, execution, data interpretation and writing the manuscript. CC and IG did the statistical analysis, data interpretation and made major contributions to writing the manuscript. MK participated in designing the database and the execution of the study. MK VP, PS, BB, and JMG participated in the study design and in drafting the manuscript. All authors critically revised and approved the final version of the manuscript and agree to be accountable for all aspects of the work

\section{Competing interests}

The authors declare that they have no competing interests.

\section{Consent for publication}

Not applicable.

\section{Ethics approval and consent to participate}

The study was approved by the institutional review boards of the Geneva Medical Association and the Geneva University Hospitals. Participants assigned to intervention group, signed consent. Consent for the control group was waived, since the HVNS routinely obtains consent for use of collected data.

\section{Author details}

${ }^{1}$ Division of Primary Care Medicine, Department of Community Medicine, Primary Care and Emergency Medicine, Geneva University Hospitals, 1, avenue Calas, Geneva 1206, Switzerland. ${ }^{2}$ Department of Ambulatory Care and Community Medicine, University of Lausanne, Lausanne, Switzerland. ${ }^{3}$ Clinical Research Centre and Division of Clinical-Epidemiology, Department of Health and Community Medicine, University of Geneva and Geneva University Hospitals, Geneva, Switzerland. ${ }^{4}$ Institution genevoise de maintien à domicile (IMAD), Carouge, Switzerland. ${ }^{5}$ Association des médecins genevois (AMG), Geneva, Switzerland. ${ }^{6}$ Cité générations, Onex, Switzerland.

\section{Received: 16 August 2016 Accepted: 11 February 2017}

\section{Published online: 14 February 2017}

\section{References}

1. Robine JM, Paccaud F. Nonagenarians and centenarians in Switzerland, 1860-2001: a demographic analysis. J Epidemiol Community Health. 2005; 59(1):31-7.

2. MacAdam M. Frameworks of Integrated Care for the Elderly: A Systematic Review. CPRN Research Report. April 2008. http://cprn.org/documents/ 49813_EN.pdf.

3. Bridges-Webb C, Giles B, Speechly C, Zurynski Y, Hiramanek N. Patients with dementia and their careers in general practice. Aust Fam Physician. 2006; 35(11):923-4.

4. Rutschmann OT, Chevalley T, Zumwald C, Luthy C, Vermeulen B, Sarasin FP. Pitfalls in the emergency department triage of frail elderly patients without specific complaints. Swiss Med Wkly. 2005:135:145-50.

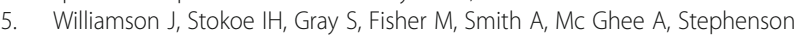
E. Old people at home their unreported needs. Lancet. 1964;1:1117-20.

6. Lubitz J, Liming Cai L, Ellen Kramarow E, Lentzner H. Health, life expectancy, and health care spending among the elderly. N Engl J Med. 2003;349(11): 1048-55.

7. Di Pollina L, Gold G, Meier D. Health care for the homebound older adult: a medical model. Mt Sinai J Med. 1993:60:488-91.

8. Gillick MR. When frail elderly adults get sick: alternatives to hospitalization. Ann Intern Med. 2014;160:201-2.

9. Temkin-Greener H, Mukamel DB. Predicting place of death in the Program of All-Inclusive Care for the Elderly (PACE): participant versus program characteristics. J Am Geriatr Soc. 2002;50:125-35.
10. Kelley AS, Ettner SL, Morrison RS, Qingling D, Sarkisian CA. Disability and decline in physical fonction associated with hospital use at the end of life. J Gen Intern Med. 2012;27(7):794-800.

11. Béland F, Hollander MJ. Integrated models of care delivery for the frail elderly: international perspectives. Gac Sanit. 2011;25(S):138-46.

12. Leichsenring K. Developing integrated health and social care services for older persons in Europe. Int J Integr Care. 2004;4:e10. Epub 2004, Sep 3.

13. Johri $\mathrm{M}$, Beland $\mathrm{F}$, Bergman $\mathrm{H}$. International experiments in integrated care for the elderly: a synthesis of the evidence. Int J Geriatr Psychiatry. 2003; 18(3):222-35.

14. Hebert R, Veil A. Monitoring the degree of implementation of an integrated delivery system. Int J Integr Care. 2004;4:e05

15. Bodenheimer T. Long-term care for frail elderly people - the On Lok model. N Engl J Med. 1999;341(17):1324-8.

16. Leutz W. Recognizing the achievements of the social health maintenance organization (SHMO) demonstration sites. J Aging Soc Policy. 1992;4(3-4):9-12.

17. Bernabei R, Landi F, Gambassi G, et al. Randomised trial of impact of model of integrated care and case management for older people living in community. BMJ. 1998:316:1348-5117.

18. Mui AC. The Program of All-Inclusive Care for the Elderly (PACE): an innovative long-term care model in the United States. J Aging Soc Policy. 2001;13(2-3):53-67.

19. Landi F, Oner G, Russo A, et al. A new model of integrated home care for the elderly: impact on hospital use. J Clin Epidemiol. 2001;54(9):968-70.

20. Tourigny A, Durand P, Bonin L, et al. Quasi-experimental study of the effectiveness of an integrated service delivery network for the frail elderly. Can J Aging. 2004;23(3):231-46.

21. Beland F, Bergman H, Lebel P, Clarfield AM, Tousignant P, Contandriopoulos AP, Dallaire L. A system of integrated care for older persons with disabilities in Canada: results from a randomized controlled trial. J Gerontol A Biol Sci Med Sci. 2006;61(4):367-73.

22. Hebert R, Raîche M, Dubois MF, et al. Impact of PRISMA, a coordination-type integrated service delivery system for frail older people in Quebec (Canada): a quasi-experimental study. J Gerontol B Psychol Sci Soc Sci. 2010;65(B):107-18.

23. De Stampa M, Vedel I, Buyck JF, et al. Impact on hospital admission of an integrated primary care model for very frail elderly patients. Arch Gerontol Geriatr. 2014;58:350-5

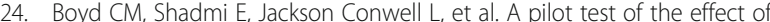
guided care on the quality of primary care experiences for multimorbid older adults. J Gen Intern Med. 2008:23(5):536-42.

25. Applegate WB, Miller ST, Graney MJ, et al. A randomized, controlled trial of a geriatric assessment unit in a community rehabilitation hospital. N Engl J Med. 1990;322(22):1572-8.

26. Rubenstein $L Z$, Stuck $A E$, Siu $A L$, Wieland D. Impacts of geriatric evaluation and management programs on defined outcomes: overview of the evidence. J Am Geriatr Soc. 1991;39(9 Pt 2):8S-16. discussion 17S-18S. Review.

27. Stuck AE, Siu AL, Wieland GD, Adams J, Rubenstein LZ. Comprehensive geriatric assessment: a meta-analysis of controlled trials. Lancet. 1993; 342(8878):1032-6.

28. Van Haastreght JCM, Diederiks JPM, van Rossum E, de Witte LP, Crebolder HFJM. Effects of preventive home visits to elderly people living in the community: a systematic review. BMJ. 2000;320:754-8.

29. Elkan R, Kendrick D, Dewey M, Hewitt M, Robinson J, Blair M, Williams D, Brummel K. Effectiveness of home based support for older people: systematic review and meta-analysis. BMJ. 2001:323:1-9.

30. Stuck AE, Egger M, Hammer A, et al. Home visits to prevent nursing home admission and functional decline in elderly people: systematic review and meta-regression analysis. JAMA. 2002;287(8):1022-8.

31. Van Hout HPJ, Jansen APD, van Marwijk HWJ, Pronk M, Frijters DF, Nijpels G. Prevention of adverse health trajectories in a vulnerable elderly population though nurse home visits: a randomized controlled trial. J Gerontol A Biol Sci Med Sci. 2010;65(7):734-42

32. Fletcher A, Price GM, Ng ESW, et al. Population-based multidimensional assessment of older people in UK general practice: a cluster randomised factorial trial. Lancet. 2004;346:1667-77.

33. Huss A, Stuck AE, Rubenstein L, Egger M, Clough-Gorr KM. Multidimensional preventive home visit programs for community-dwelling older adults: A systematic review and meta-analysis of randomized controlled trials. J Gerontol A Biol Sci Med Sci. 2008;63A(3):298-307.

34. Beswick AD, Rees K, Dieppe P, Ayis S, Gooberman-Hill R, Horwood J, Ebranhim S. Complex interventions to improve physical function and maintain independent 
living in elderly people: a systematic review and meta-analysis. Lancet. 2008; 371(9614):725-35.

35. Rosenberg T. Acute hospital use, nursing home placement, and mortality in frail community-dwelling cohort managed with primary integrated interdisciplinary elder care at home. J Am Geriatr Soc. 2012;60:1340-6.

36. Metzelthin SF, van Rossum E, de Witt LP, et al. Effectiveness of interdisciplinary primary care approach to reduce disability in community dwelling frail older people: cluster randomised controlled trial. BMJ. 2013; 347:f5264. doi:10.1136/bmj.f5264.

37. Fries BE, Hawes C, Morris JN, Phillips CD, Mor V, Park PS. Effect of the national resident assessment instrument on selected health conditions and problems. J Am Geriatr Soc. 1997;45(8):994-1001.

38. Landi F, Tua E, Onder G, Carrara B, et al. Minimun data set for home care: valid instrument to assess frail older people living in the community. Med Care. 2000:38:1184-90.

39 Hirdes JP. Addressing the health needs of frail elderly people: Ontario's experience with an integrated health information system. Age Ageing. 2006;35:329-31.

40 Folstein MF, Folstein SE, Mchugh PR. Mini mental state : a practical method for grading the cognitive state of patients for the clinicians. J Psychiatr Res. 1975;12:189-98.

41 Yesavage JA, Brink TL, Rose TL, et al. Development and validation of a geriatric depression screening scale: a preliminary report. J Psychiatr Res. 1982;17(1):37-49.

42 Katz S. Progress in development of the index of ADL. J Gerontol. 1970;10(1): 20-30.

43 Lawton MP, Brody EM. Assessment of older people: self-maintaining Gerontologist. 1969;9:179-85.

44 Podsiadlo D, Richardson S. The timed "Up \& Go": a test of basic functional mobility for frail elderly persons. J Am Geriatr Soc. 1991;39(2):142-8.

45 Vellas BJ, Rubenstein LZ, Ousset PJ, et al. One-leg standing balance and functional status in a population of 512 community-living elderly persons. Aging (Milano). 1997;9(1-2):95-8.

46 Guigoz Y, Vellas BJ, Garry PJ. Assessing the nutritional status of the elderly: the mini nutritional assessment as part of the geriatric evaluation. Nutr Rev. 1996;54(1):S59-65.

47 Di Pollina L, Gillabert C, Picard-Kossosvky M. Integrated care systems for elderly people. Rev Med Suisse. 2008;4:2044-7.

48 Gray RJ. A class of K-sample tests for comparing the cumulative incidence of a competing risk. Ann Stat. 1988;16:1141-54.

49 Fine JP, Gray RJ. A proportional hazards model for the sub distribution of a competing risk. JASA. 1999;94:496-509.

50 https://www.ge.ch/ems/chiffres_cles.asp. Accessed 12 Nov 2013

51 Fried LP, Tangen CM, Walston J, Newman AB, Hirsch C, Gottdiener J, et al. Frailty in older adults: evidence for a phenotype. J Gerontol A Biol Sci Med Sci. 2001;56:M146-56.

\section{Submit your next manuscript to BioMed Central and we will help you at every step:}

- We accept pre-submission inquiries

- Our selector tool helps you to find the most relevant journal

- We provide round the clock customer support

- Convenient online submission

- Thorough peer review

- Inclusion in PubMed and all major indexing services

- Maximum visibility for your research

Submit your manuscript at www.biomedcentral.com/submit 